Pacific Journal of Mathematic 


\section{ON THE DETERMINATION OF NUMBERS BY THEIR SUMS OF A FIXED ORDER}

\section{J. L. Selfridge and E. G. Straus}

1. Introduction. We wish to treat the following problem (suggested by a problem of L. Moser [2]) :

Let $\{x\}=\left\{x_{1}, \cdots, x_{n}\right\}$ be a set of complex numbers (if one is interested in generality, one may consider them elements of an algebraically closed field of characteristic zero) and let $\{\sigma\}=\left\{\sigma_{1}, \cdots, \sigma_{\left(\begin{array}{c}n \\ s\end{array}\right)}\right\}$ be the set of sums of $s$ distinct elements of $\{x\}$. To what extent is $\{x\}$ determined by $\{\sigma\}$ and what sets can be $\{\sigma\}$ sets ?

In $\S 2$ we answer this question for $s=2$. In $\S 3$ we treat the question for general $s$.

\section{The case $s=2$.}

THEOREM 1. If $n \neq 2^{k}$ then the first $n$ elementary symmetric functions of $\{\sigma\}$ can be prescribed arbitrarily and they determine $\{x\}$ uniquely.

Proof. Instead of the elementary symmetric functions we consider the sums of powers, setting

$$
\sum_{k}=\sum_{i=1}^{\left(\begin{array}{c}
n \\
2
\end{array}\right)} \sigma_{i}^{k}, \quad S_{k}=\sum_{i=1}^{n} x_{i}^{k}
$$

Then

$$
\begin{aligned}
\sum_{k} & =\sum_{i=1}^{\left(\begin{array}{l}
n \\
2
\end{array}\right)} \sigma_{i}^{k}=\sum_{1 \leq i_{1}<i_{2} \leq n}\left(x_{i_{1}}+x_{i_{2}}\right)^{k}=\frac{1}{2} \sum_{\substack{i_{1}, i_{2}=1 \\
i_{1} \neq i_{2}}}^{n}\left(x_{i_{1}}+x_{i_{2}}\right)^{k} \\
& =\frac{1}{2}\left(\sum_{i_{1}, i_{2}=1}^{n}\left(x_{i_{1}}+x_{i_{2}}\right)^{k}-\sum_{i=1}^{n}\left(2 x_{i}\right)^{k}\right) .
\end{aligned}
$$

Expanding the binomials and collecting like powers we obtain

$$
\begin{aligned}
\sum_{k} & =\frac{1}{2}\left(\sum_{l=0}^{k}\left(\begin{array}{l}
k \\
l
\end{array}\right) S_{l} S_{k-l}-2^{k} S_{k}\right) \\
& =\frac{1}{2}\left(2 n-2^{k}\right) S_{k}+\frac{1}{2} \sum_{l=1}^{k-1}\left(\begin{array}{l}
k \\
l
\end{array}\right) S_{l} S_{k-l}
\end{aligned}
$$

Thus, since the coefficient of $S_{k}$ does not vanish, we can solve reReceived May 16, 1958. 
cursively for $S_{k}$ in terms of $\sum_{1}, \cdots, \sum_{k}$. In particular $\sum_{1}, \cdots, \sum_{n}$ determine $S_{1}, \cdots, S_{n}$ - and hence $x_{1}, \cdots, x_{n}$-uniquely.

THEOREM 2. If $n=2^{k}$ then $\sum_{1}, \cdots, \sum_{k+1}$ must satisfy a certain algebraic equation and $\{\sigma\}$ will not always determine $\{x\}$.

Proof. Equation (1) for $\sum_{k+1}$ yields

$$
\sum_{k+1}=\frac{1}{2} \sum_{l=1}^{k}\left(\begin{array}{c}
k+1 \\
l
\end{array}\right) S_{l} S_{k+1-l}
$$

where $S_{1}, \cdots, S_{k}$ are expressed by (1) as polynomials in $\sum_{1}, \cdots, \Sigma_{k}$.

To prove the second part of the theorem we proceed by induction.

Assume there are two different sets $\left\{x_{1}, \cdots, x_{2^{k-1}}\right\},\left\{y_{1}, \cdots, y_{2^{k-1}}\right\}$ which have the same $\{\sigma\}$. Then consider the two sets

$$
\begin{aligned}
& \{X\}=\left\{x_{1}+a, \cdots, x_{2^{k-1}}+a, y_{1}, \cdots, y_{2^{k-1}}\right\} \\
& \{Y\}=\left\{x_{1}, \cdots, x_{2^{k-1}}, y_{1}+a, \cdots, y_{2^{k-1}}+a\right\} .
\end{aligned}
$$

Clearly every sum of two elements of $\{X\}$ is either $\sigma_{i}$ or $\sigma_{i}+2 a$ or $x_{i}+y_{j}+a$ and the same holds for the sum of two elements of $\{Y\}$.

The sets $\{X\},\{Y\}$ will clearly be different for some $a$. To show that they are different for any $a \neq 0$, rearrange $\{x\}$ and $\{y\}$ so that $x_{i}=y_{i} ; i=1,2, \cdots, m ; m \geq 0$, and $x_{j} \neq y_{k}$ for $j, k>m$. Then since $y_{i}+a=x_{i}+a ; i=1,2, \cdots, m$, the sets $\{X\}$ and $\{Y\}$ will be different if $\left\{x_{j} \mid j>m\right\}$ is different from $\left\{x_{j}+a \mid j>m\right\}$. But this is clear for any $a \neq 0$.

Since $\{\sigma\}$ clearly does not determine $\{x\}$ for $n=2$ the proof is complete.

In a sense we have completed the answer of the question raised in the introduction for $s=2$, however there remain some unanswered questions in case $n=2^{k}$.

1. If $\{\sigma\}$ does not determine $\{x\}$ can there be more than two sets giving rise to same $\{\sigma\}$ ?

The answer is trivially "yes" for $k=0,1$ and is " no" for $k=2$. It seems probable that the answer is " no" for all $k \geq 2$, however we can see no simple way of proving this.

2. For what values of $n$ does there exist for all (real) $\{x\}$ a transformation $y_{i}=f_{i}\left(x_{1}, \cdots, x_{n}\right)$, different from a permutation, so that $\{x\}$ and $\{y\}$ give rise to the same $\{\sigma\}$ ?

This question was suggested by T. S. Motzkin who gave the answer for $s=2$. 
LEMmA 1. If $n>s$ and the above functions $f_{i}$ exist then they are linear.

Proof. The sets $\{y\},\{x\}$ are connected by a system of equations

$$
y_{i_{1}}+\cdots+y_{i_{s}}=x_{j_{1}}+\cdots+x_{j_{s}} .
$$

Here the indices $i_{1}, \cdots, i_{s}$ are themselves functions of $\{x\}$. However, since they assume only a finite set of values, there exists a somewhere dense set of $\{x\}$ for which the indices are constant. We restrict our attention to that set. Let $\Delta_{k}^{(h)} y_{i}=f\left(x_{1}, \cdots, x_{k}+h, \cdots, x_{n}\right)-f_{i}\left(x_{1}, \cdots\right.$, $x_{k}, \cdots, x_{n}$ ) then we obtain

$$
\Delta_{k}^{(h)} y_{i_{1}}+\cdots+\Delta_{k}^{(h)} y_{i_{s}}=0 \text { or } h .
$$

If we let $u_{i}$ be the difference of $\Delta_{k}^{(h)} y_{i}$ for two different sets of values of $\{x\}$ then, since the right-hand side of (3) is independent of the choice of $\{x\}$, we obtain

$$
u_{i_{1}}+\cdots+u_{i_{s}}=0 .
$$

Summation over all sets $\left\{i_{1}, \cdots, i_{s}\right\} \subset\{1, \cdots, n\}$ yields

$$
u_{1}+u_{2}+\cdots+u_{n}=0 \text {. }
$$

Now let $t$ be the least positive integer so that $u_{i_{1}}+\cdots+u_{i_{t}}=0$ for all $\left\{i_{1}, \cdots, i_{t}\right\} \subset\{1, \cdots, n\}$. Then $t \mid n$, for $n=m t+r$ with $0<r<t$ implies

$$
u_{i_{1}}+\cdots+u_{i_{r}}=u_{1}+u_{2}+\cdots+u_{n}-\Sigma\left(u_{j_{1}}+\cdots+u_{j_{t}}\right)=0
$$

for all $\left\{i_{1}, \cdots, i_{r}\right\} \subset\{1, \cdots, n\}$, contrary to hypothesis.

Since $n>s \geq t$ we must have $n \geq 2 t$. If $t>1$ then

$$
u_{j}=-\left(u_{i_{1}}+\cdots+u_{i_{t-1}}\right) \text { for every } j \notin\left\{i_{1}, \cdots, i_{t-1}\right\} \text {. }
$$

But there are more than $t$ such $j$, say $j_{1}, \cdots, j_{t}$. Hence

$$
u_{j_{1}}+\cdots+u_{f_{t}}=-t\left(u_{i_{1}}+\cdots+u_{i_{t-1}}\right)=0
$$

or $u_{i_{1}}+\cdots+u_{i_{t-1}}=0$ for every $\left\{i_{1}, \cdots, i_{t-1}\right\} \subset\{1, \cdots, n\}$ contrary to hypothesis. Thus $t=1$ and

$$
u_{1}=u_{2}=\cdots=u_{n}=0 \text {. }
$$

In other words $\Delta_{k}^{(h)} y_{i}=a_{i k}^{(h)}=$ const. Thus $\Delta_{k}^{\left(h_{1}\right)} y_{i}+\Lambda_{k}^{(h)} y_{i}=\Delta_{k}^{\left(h_{1}+h_{2}\right)} y_{i}$ so that $a_{i k}^{(h)}=a_{i k} h$ and

$$
y_{i}=\sum_{k} a_{i k} x_{k}
$$


THEOREM 3. If $n>s$ and there exists a nontrivial transformation $y_{i}=f_{i}\left(x_{1}, \cdots, x_{n}\right)$ which preserves $\{\sigma\}$ then $n=2 s$ and the transformation is linear with matrix (up to permutations)

$$
\left(\begin{array}{cccc}
-\frac{s-1}{s} & \frac{1}{s} & \cdots & \frac{1}{s} \\
\frac{1}{s} & -\frac{s-1}{s} & \ldots & \frac{1}{s} \\
\vdots & \vdots & & \vdots \\
\frac{1}{s} & \frac{1}{s} & \cdots & s-1 \\
\frac{1}{s}
\end{array}\right)
$$

Proof. We know by Lemma 1 that the transformation must be linear. Let $y_{i}=\sum_{k} a_{i k} x_{k}$ then

$$
y_{i_{1}}+\cdots+y_{i_{s}}=\sum_{k}\left(a_{i_{1} k}+\cdots+a_{i_{s} k}\right) x_{k}=x_{j_{1}}+\cdots+x_{j_{s}} .
$$

Hence, for fixed $k$, we have

$$
a_{i_{1} k}+\cdots+a_{i_{s} k}=\left\{\begin{array}{l}
0 \text { for }\left(\begin{array}{c}
n-1 \\
s
\end{array}\right) \text { sets }\left\{i_{1}, \cdots, i_{s}\right\} \\
1 \text { for }\left(\begin{array}{c}
n-1 \\
s-1
\end{array}\right) \text { sets }\left\{i_{1}, \cdots, i_{s}\right\} .
\end{array}\right.
$$

Since $n>s$ two elements $a_{i k}, a_{j k}$ in the same column satisfy

$$
a_{i k}+a_{i_{1} k}+\cdots+a_{i_{s-1} k}=0 \text { or } 1 ; a_{f k}+a_{i_{1} k}+\cdots+a_{i_{s-1} k}=0 \text { or } 1
$$

where $\left\{i_{1}, \cdots, i_{s-1}\right\} \subseteq\{1, \cdots, n\}-\{i, j\}$.

\section{Hence}

$$
a_{i k}=a_{j k} \text { or } a_{i k}=a_{j k} \pm 1 .
$$

Let the two values assumed by terms in the $k$ th column be $a_{k}$ and $1+a_{k}$. From (6) we see that both values must occur. On the other hand if both $a_{k}$ and $1+a_{k}$ would occur more than once then $\max \left(a_{i_{1} k}+\cdots+a_{i_{s} k}\right)-\min \left(a_{i_{1} k}+\cdots+a_{i_{s} k}\right) \geq 2$ in contradiction to (7).

If $1+a_{k}$ is assumed only once, say $a_{k k}=1+a_{k}$, then $0=s a_{k}$ or

$$
a_{i k}=\left\{\begin{array}{ll}
1 & i=k \\
0 & i \neq k
\end{array} .\right.
$$

According to (6) we have

$$
\sum_{k=1}^{n}\left(a_{i_{1} k}+\cdots+a_{i_{s} k}\right)=s \quad\left\{i_{1}, \cdots, i_{s}\right\} \subset\{1, \cdots, n\} .
$$

We now repeat the argument that led to equation (8). Since $n>\mathrm{s}$ 
we can write for any pair $(i, j)$

$$
\sum_{k=1}^{n}\left(\alpha_{i_{1} k}+\cdots+a_{i_{s-1} k}\right)+\sum_{k=1}^{n} \alpha_{i k}=\sum_{k=1}^{n}\left(\alpha_{i_{1} k}+\cdots+a_{i_{s-1} k}\right)+\sum_{k=1}^{n} a_{j k}=s
$$

where $\left\{i_{1}, \cdots, i_{s-1}\right\} \subset\{1, \cdots, n\}-\{i, j\}$. Hence $\sum_{k=1}^{n} a_{i k}=\sum_{k=1}^{n} a_{j k}$ and according to (10), $s \sum_{k=1}^{n} a_{i k}=s$ so that

$$
\sum_{k=1}^{n} a_{i k}=1 \quad i=1, \cdots, n .
$$

Combining (9) and (11) we obtain

$$
a_{k j}=\left\{\begin{array}{ll}
1 & j=k \\
0 & j \neq k
\end{array} .\right.
$$

In other words, every column contains 0 and therefore $a_{k}=0$ for $k=1, \cdots, n$. Thus the transformation is a permutation.

The only nontrivial case arises therefore if the value $a_{k}$ occurs only once, say $a_{k k}=a_{k}$. Then $s-1+s a_{k}=0$ and

$$
a_{i k}=\left\{\begin{array}{ll}
-(s-1) / s & i=k \\
1 / s & i \neq k
\end{array} .\right.
$$

Combining (11) and (13) we obtain

$$
\sum_{k=1}^{n} \sum_{i=1}^{n} a_{i k}=n=\frac{n(n-1)}{s}-n \frac{s-1}{s}=\frac{n}{s}(n-s)
$$

and hence $n=2 s$. It is now clear from (11) that each row and column contains exactly one term - $(s-1) / s$ and that the matrix (up to permutation) is the one given in the theorem.

3. General $s$. The procedure which led to Theorem 1 can be generalized. First we define, for every $s$, a function which is a polynomial in $n, 2^{k}, 3^{k}, \cdots, s^{k}$. Let

$$
f(n, k)=\frac{1}{s} \sum_{P}(-1)^{s-\iota} n^{\iota-1} \sum_{i=1}^{r} a_{i} i^{k}
$$

where the outer summation is over all permutations $P$ on $s$ marks, each permutation being composed of $a_{i} i$-cycles $i=1, \cdots, r$, and $t=$ $a_{1}+\cdots+a_{r}$. Thus

(16) $f(n, k)=n^{s-1}-\frac{1}{2}(s-1)\left(2^{k}+s-2\right) n^{s-2}+(s-1)(s-2)\left[\frac{1}{3}\left(3^{k}+s-3\right)\right.$

$$
\begin{aligned}
& \left.+\frac{1}{8}(s-3)\left(2^{k+1}+s-4\right)\right] n^{s-3}-\cdots+(-1)^{s}(s-1) !\left(\sum_{i=1}^{s-1} \frac{i^{k-1}}{s-i}\right) n \\
& -(-1)^{s}(s-1) ! s^{k-1} .
\end{aligned}
$$


THEOREM 4. For every s consider the system of Diophantine equations $f(n, k)=0 \quad k=1,2, \cdots, n$. If $n$ satisfies none of these then the first $n$ elementary symmetric functions of $\{\sigma\}$ can be prescribed arbitrarily and they determine $\{x\}$ uniquely. If $f(n, k)=0$, then the first $k$ elementary symmetric functions of $\{\sigma\}$ must satisfy an algebraic equation.

Proof. In the notation of Theorem 1 we have

$$
\sum_{k} \underset{1 \leq i_{1}<\cdots<i_{s} \leq n}{ }\left(x_{i_{1}}+x_{i_{2}}+\cdots+x_{i_{s}}\right)^{k}=\frac{1}{s !} \sum_{D(s)}\left(x_{i_{1}}+\cdots+x_{i_{s}}\right)^{k}
$$

where by $D(t)$ is meant summation over all sets of subscripts $i_{\text {f }}$ at least $t$ of which are distinct. Hence

$$
\begin{gathered}
s ! \sum_{k}=\sum_{D(s-1)}\left(x_{i_{1}}+\cdots+x_{i_{s}}\right)^{k}-\left(\begin{array}{l}
s \\
2
\end{array}\right) \sum_{D(s-1)}\left(2 x_{i_{1}}+x_{i_{2}}+\cdots+x_{i_{s-1}}\right)^{k} \\
=\sum_{D(s-2)}\left(x_{i_{1}}+\cdots+x_{i_{s}}\right)^{k}-\left(\begin{array}{l}
s \\
2
\end{array}\right) \sum_{D(s-2)}\left(2 x_{i_{1}}+x_{i_{2}}+\cdots+x_{i_{s-1}}\right)^{k} \\
+2\left(\begin{array}{l}
s \\
3
\end{array}\right) \sum_{D(s-2)}\left(3 x_{i_{1}}+x_{i_{2}} \cdots+x_{i_{s-2}}\right)^{k}+3\left(\begin{array}{l}
s \\
4
\end{array}\right) \sum_{D(s-2)}\left(2 x_{i_{1}}+2 x_{i_{2}}+x_{i_{3}}+\cdots+x_{i_{s-2}}\right)^{k} .
\end{gathered}
$$

Continue cancelling terms until each summation is over $D(1)$. The coefficient of $\sum\left(m_{1} x_{i_{1}}+\cdots+m_{t} x_{i_{t}}\right)^{k}$ is just $(-1)^{s-t}$ times the number of permutations on $s$ marks which are conjugate to one having cycles of length $m_{1}, \cdots, m_{t}$. This can be shown by a method quite similar to that used by Frobenius [1]. Hence we may write

$$
s ! \sum_{k}=\sum_{P}(-1)^{s-t} \sum_{D(1)}\left(m_{1} x_{i_{1}}+\cdots+m_{t} x_{i_{t}}\right)^{k}
$$

where the outer summation is over all permutations $P$ on $s$ marks, and $m_{1}, \cdots, m_{t}$ are the lengths of the cycles of $P$. Now from the multinomial expansion we have

$$
\sum_{D(1)}\left(m_{1} x_{i_{1}}+\cdots+m_{t} x_{i_{t}}\right)^{k}=\sum_{\substack{l_{1}+\underbrace{}_{i+l_{t}}=k \\ i_{i} \geq 0}} \frac{k !}{l_{1} ! \cdots l_{t} !} m_{1}^{l_{1}} \cdots m_{t}^{l_{t}} S_{l_{1}} \cdots S_{l_{t}}
$$

and the coefficient of $S_{k}$ is $\left(m_{1}^{k}+\cdots+m_{t}^{k}\right) S_{0}^{t-1}$. Substituting in (18) and using (15), we obtain

$$
(s-1) ! \sum_{k}=f(n, k) S_{k}+\cdots
$$

where the terms indicated by dots do not involve $S_{k}$. Thus, if $f(n, k) \neq 0$ for $k=1, \cdots, n$, then (19) can be solved recursively for $S_{1}, \cdots, S_{n}$ in terms of $\Sigma_{1}, \cdots, \Sigma_{n}$.

On the other hand, if $f(n, k)=0$ and $f(n, j) \neq 0$ for $j=1, \cdots, k-1$ then (17) expresses $\sum_{k}$ as a polynomial in $S_{1}, \cdots, S_{k-1}$ which in turn are polynomials in $\Sigma_{1}, \cdots, \Sigma_{k-1}$. 
Corollary. If $f(n, k)=0$ then $n$ divides $(s-1) ! s^{n-1}$.

Thus $\{x\}$ will always be determined by $\{\sigma\}$ if $s$ is less then the greatest prime factor of $n$.

Example 1. $s=3$. Here (18) becomes

$$
6 \sum_{k}=\sum_{i_{1}, i_{2}, i_{3}=1}^{n}\left(x_{i_{1}}+x_{i_{2}}+x_{i_{3}}\right)^{k}-3 \sum_{i_{1}, i_{2}=1}^{n}\left(2 x_{i_{1}}+x_{i_{2}}\right)^{k}+2 \sum_{i=1}^{n}\left(3 x_{i}\right)^{k} .
$$

Expanding and collecting the coefficient of $S_{k}$, we get

$$
f(n, k)=n^{2}-\left(2^{k}+1\right) n+2 \cdot 3^{k-1} .
$$

This has obvious zeros at $n=1, k=1 ; n=2, k=1,2 ; n=3, k=2,3$. Also, as we know from Theorem 3 , there are zeros at $n=6, k=3,5$. For all these values of $n$ the set $\{\sigma\}$ does not, in general, determine $\{x\}$ uniquely.

In addition we observe that $f(n, k)=0$ has the solutions $n=27$, $k=5,9$ and $n=486, k=9$. We do not know whether for these values of $n$ the set $\{\sigma\}$ determines $\{x\}$ uniquely or not. However we do know that these are the only cases left in doubt.

TheOREM 5. If $s=3$ then $f(n, k)=0$ has solutions only for $k=$ $1,2,3,5,9$.

Proof. If $f(n, k)=0$ then

$$
n=2^{a} \cdot 3^{b} \text { with } a=0 \text { or } 1 .
$$

Substituting (20) in $f(n, k)=0$ we obtain

$$
2^{a} \cdot 3^{b}+2^{1-a} 3^{k-b-1}=2^{k}+1 .
$$

Let $n$ be the smaller zero of $f(n, k)$ for a fixed $k$. Then the other zero is $n^{\prime}=2^{1-a} 3^{k-b-1}$ and $b \leq k-b-1$. Hence

$$
2^{k} \equiv-1\left(\bmod 3^{b}\right)
$$

and since 2 is a primitive root of $3^{b}$,

$$
k \equiv 3^{b-1}\left(\bmod 2 \cdot 3^{b-1}\right) .
$$

But by (21) we have

$$
3^{k-b-1} \leq 2^{k}<3^{2 k / 3} \text { or } k<3(b+1)
$$

so that

$$
3^{b-1} \leq k<3(b+1) \text { and hence } b<4
$$


If $b=3$ then $k \equiv 9(\bmod 18)$ and $k<12$ so $k=9$.

If $b=2$ then $k \equiv 3(\bmod 6)$ and $k<9$ so $k=3$.

If $b=1$ then $k \equiv 1(\bmod 2)$ and $k<6$ so $k=1,3,5$.

If $b=0$ then $k<3$.

EXAmple 2. $s=4$. Here (18) becomes

$$
\begin{aligned}
24 \sum_{k} & =\sum_{i_{1}, i_{2}, i_{3}, i_{4}}\left(x_{i_{1}}+x_{i_{2}}+x_{i_{3}}+x_{i_{4}}\right)^{k} \\
& -6 \sum_{i_{1}, i_{2}, i_{3}}\left(2 x_{i_{1}}+x_{i_{2}}+x_{i_{3}}\right)^{k}+8 \sum_{i_{1}, i_{2}}\left(3 x_{i_{1}}+x_{i_{2}}\right)^{k} \\
& +3 \sum_{i_{1}, i_{2}}\left(2 x_{i_{1}}+2 x_{i_{2}}\right)^{k}-6 \sum_{i}\left(4 x_{i}\right)^{k} .
\end{aligned}
$$

Hence $f(n, k)=0$ becomes

$$
n^{3}-3\left(2^{k-1}+1\right) n^{2}+\left(2\left(3^{k}+1\right)+3 \cdot 2^{k-1}\right) n-3 \cdot 2^{2 k-1}=0 .
$$

We first note that this has solutions $n=1, k=1 ; n=2, k=1,2$; $n=3, k=1,2,3 ; n=4, k=2,3,4 ; n=8, k=3,5,7$. For these values of $n$, the set $\{\sigma\}$ does not generally determine $\{x\}$. When $n=12, k=6$ is a solution, and this case is left in doubt.

THEOREM 6. If $s=4$ then $f(n, k)=0$ has solutions only for $n=$ $1,2,3,4,8,12$.

Proof. Let $n=3^{a} \cdot 2^{b}$ where $a=0$ or 1 . Now if $n \geq 3\left(2^{k-1}+1\right)$ then $2 \cdot 3^{k} n>3^{k+1} \cdot 2^{k}>3 \cdot 2^{2 k-1}$ and the left side of (25) is positive. Hence $n<3\left(2^{k-1}+1\right)<2^{k+1}$ if $k>3$ and so $b \leq k$. (For $k \leq 3$ we have listed all solutions of $(25))$. If $k$ is even then $2\left(3^{k}+1\right) \equiv 4(\bmod 8)$ and if $k \geq 4$ then $8 n$ divides the other terms unless $b \leq 2$. Similarly if $k$ is odd then $2\left(3^{k}+1\right) \equiv 8(\bmod 16)$ and if $k \geq 5$ then $b \leq 3$. So $b \leq 3$ in all cases. Now suppose $a=1$. Then (25) becomes

$$
2 n-3 \cdot 2^{2 k-1} \equiv 0(\bmod 9)
$$

or

$$
2^{b+1} \equiv 2^{2 k-1} \equiv 2(\bmod 3)
$$

and $b$ is even. Thus $n$ must be $1,2,3,4,8$, or 12 . It is easy to check that none of these is a root for $k>7$.

The corollary to Theorem 4 shows that exceptional pairs $(s, n)$ are in a certain sense quite rare. Of course it is trivial to remark that if $(s, n)$ is exceptional, then $(n-s, n)$ is exceptional. Hence the remarks for $s=2$ apply equally well to $s=n-2$ and we obtain the exceptional pairs $(6,8),(14,16),(30,32), \cdots$. But there are other cases with $n>2 s$ which our method leaves in doubt. 
THEOREM 7. We can construct arbitrarily large values of $s$ such that $f(n, k)=0$ for some $n>2 s$.

Proof. If $n<s$ then $\sum_{k}=0$ but $S_{1}, \cdots, S_{n}$ may be prescribed arbitrarily. Hence the coefficient of $S_{k}$ in the expansion of $\sum_{k}$ must be zero if $k \leq n$. If $n=s$ then $\sum_{k}=S_{1}^{k}$ but $S_{2}, \cdots, S_{n}$ may be prescribed arbitrarily. Hence $n=s$ is a zero of $f(n, k)$ for $k=2, \cdots, n$. Thus $f(n, 1)=\prod_{i=1}^{s-1}(n-i) ; f(n, 2)=\prod_{i=2}^{s}(n-i)$ and $f(n, 3)=(n-2 s) \prod_{i=3}^{s}(n-i)$. If we divide $f(n, 4)$ by its known factors then we obtain for $s>2$

$$
f(n, 4)=\left[n^{2}-(6 s-1) n+6 s^{2}\right] \prod_{i=4}^{s}(n-i)
$$

and the equation

$$
n^{2}-(6 s-1) n+6 s^{2}=0
$$

can be rewritten

$$
(2 n-6 s+1)^{2}-3(2 s-1)^{2}=-2 .
$$

The Pell equation $u^{2}-3 v^{2}=-2$ has the general solution

$$
u+v \sqrt{3}= \pm(1+\sqrt{3})(2+\sqrt{ } 3)^{r} \quad r=0, \pm 1, \cdots .
$$

Since $u$ and $v$ are odd, $n$ and $s$ are integers. It is interesting that all positive solutions are obtained in the following simple way. When $k=4,(s, n)=(2,8)$ is a solution. Hence $(6,8)$ is a solution and putting $s=6$ in $(27)$ yields $(6,27)$. Continuing in this way, we obtain $(21,27)$, (21, 98), $(77,98),(77,363), \cdots$.

In a similar manner we obtain for $s>3$

$$
f(n, 5)=\left[n^{2}-(12 s-5) n+12 s^{2}\right](n-2 s) \prod_{i=5}^{s}(n-i)
$$

and all integer roots of the quadratic factor may be obtained with the aid of the general solution of the Pell equation $u^{2}-6 v^{2}=75$. Or we could start with $(2,16)$ and obtain successively $(14,147),(133,1444), \cdots$. Starting with $(3,27)$ yields $(24,256),(232,2523), \cdots$.

4. Concluding remarks. If we let $\{\tau\}=\left\{\tau_{1}, \cdots, \tau_{n s}\right\}$ be the set of sums of $s$ not necessarily distinct elements of $\{x\}$, then $\{x\}$ is always determined by $\{\tau\}$. A method similar to the proof of Theorem 4 applies with the coefficient of $S_{k}$ always positive. Alternatively, if the $x_{i}$ are real, $x_{1} \leq x_{2} \leq \cdots \leq x_{n}$, we may determine them successively by a simple induction procedure.

Our method is applicable to the case of weighted sums $\sigma_{i_{1} \cdots i_{s}}=$ 
$\sum_{j=1}^{s} a_{j} x_{i_{j}}$. The resulting Diophantine equations will however be of a rather different nature. Thus, if the $a_{\text {, }}$ are all distinct then the analogue to $f(n, k)=0$ is

$$
\left(a_{1}^{k}+a_{2}^{k}+\cdots+a_{s}^{k}\right) n^{s-1}=0 .
$$

In other words the uniqueness condition is independent of $n$ and depends on the $a_{i}$ alone. For example if $a_{1}+a_{2}+\cdots+a_{s}=0$ then $\{\sigma\}$ remains unchanged if we add the same constant to all $x$. It is not as easy to see what happens if (29) holds for some $k>1$.

\section{REFERENCES}

1. G. Frobenius, Ueber die Charaktere der symmetrischen Gruppe, S.-B. Preuss. Akad. Wiss. Berlin, (1900), 516-534.

2. L. Moser, Problem E 1248, Amer. Math. Monthly (1957), 507.

UNiversity of CALIFornia, Los ANGeles 


\section{PACIFIC JOURNAL OF MATHEMATICS}

\section{EDITORS}

\section{David Gilbarg}

Stanford University

Stanford, California

\section{R. A. Beaumont}

University of Washington

Seattle 5, Washington

\author{
A. L. Whiteman
}

University of Southern California Los Angeles 7, California

E. G. Straus

University of California

Los Angeles 24, California

\section{ASSOCIATE EDITORS}

\author{
E. F. BECKENBACH \\ C. E. BURGESS \\ M. HALL \\ E. HEWITT
}
A. HORN
V. GANAPATHY IYER
R. D. JAMES
M. S. KNEBELMAN
L. NACHBIN
I. NIVEN
T. G. OSTROM
H. L. ROYDEN

M. M. SCHIFFER

G. SZEKERES

F. WOLF

K. YOSIDA

\section{SUPPORTING INSTITUTIONS}

\author{
UNIVERSITY OF BRITISH COLUMBIA \\ CALIFORNIA INSTITUTE OF TECHNOLOGY \\ UNIVERSITY OF CALIFORNIA \\ MONTANA STATE UNIVERSITY \\ UNIVERSITY OF NEVADA \\ OREGON STATE COLLEGE \\ UNIVERSITY OF OREGON \\ OSAKA UNIVERSITY \\ UNIVERSITY OF SOUTHERN CALIFORNIA
}

\author{
STANFORD UNIVERSITY \\ UNIVERSITY OF TOKYO \\ UNIVERSITY OF UTAH \\ WASHINGTON STATE COLLEGE \\ UNIVERSITY OF WASHINGTON \\ * * * * \\ AMERICAN MATHEMATICAL SOCIETY \\ CALIFORNIA RESEARCH CORPORATION \\ HUGHES AIRCRAFT COMPANY \\ THE RAMO-WOOLDRIDGE CORPORATION
}

Mathematical papers intended for publication in the Pacific Journal of Mathematics should be typewritten (double spaced), and the author should keep a complete copy. Manuscripts may be sent to any one of the four editors. All other communications to the editors should be addressed to the managing editor, E. G. Straus at the University of California, Los Angeles 24, California.

50 reprints per author of each article are furnished free of charge; additional copies may be obtained at cost in multiples of 50 .

The Pacific Journal of Mathematics is published quarterly, in March, June, September, and December. The price per volume (4 numbers) is $\$ 12.00$; single issues, $\$ 3.50$. Back numbers are available. Special price to individual faculty members of supporting institutions and to individual members of the American Mathematical Society: $\$ 4.00$ per volume; single issues, $\$ 1.25$.

Subscriptions, orders for back numbers, and changes of address should be sent to Pacific Journal of Mathematics, 2120 Oxford Street, Berkeley 4, California.

Printed at Kokusai Bunken Insatsusha (International Academic Printing Co., Ltd.), No. 6, 2-chome, Fujimi-cho, Chiyoda-ku, Tokyo, Japan.

PUBLISHED BY PACIFIC JOURNAL OF MATHEMATICS, A NON-PROFIT CORPORATION

The Supporting Institutions listed above contribute to the cost of publication of this Journal, but they are not owners or publishers and have no responsibility for its content or policies. 


\section{Pacific Journal of Mathematics}

\section{Vol. 8, No. $4 \quad$ June, 1958}

Richard Arens, The maximal ideals of certain functions algebras ........ 641

Glen Earl Baxter, An operator identity ........................... 649

Robert James Blattner, Automorphic group representations ........... 665

Steve Jerome Bryant, Isomorphism order for Abelian groups ............ 679

Charles W. Curtis, Modules whose annihilators are direct summands...... 685

Wilbur Eugene Deskins, On the radical of a group algebra ............ 693

Jacob Feldman, Equivalence and perpendicularity of Gaussian

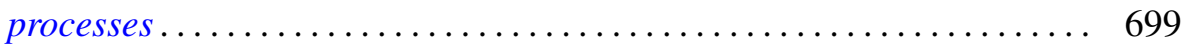

Marion K. Fort, Jr. and G. A. Hedlund, Minimal coverings of pairs by triples....................................... 709

I. S. Gál, On the theory of $(m, n)$-compact topological spaces ......... 721

David Gale and Oliver Gross, A note on polynomial and separable games........................................ 735

Frank Harary, On the number of bi-colored graphs ............... 743

Bruno Harris, Centralizers in Jordan algebras ................... 757

Martin Jurchescu, Modulus of a boundary component ............... 791

Hewitt Kenyon and A. P. Morse, Runs . . . . . . . . . . . . . . . . . . . . . . 811

Burnett C. Meyer and H. D. Sprinkle, Two nonseparable complete metric

spaces defined on $[0,1] \ldots \ldots \ldots \ldots \ldots \ldots \ldots \ldots \ldots \ldots \ldots . \ldots . \ldots . \ldots . \ldots 25$

M. S. Robertson, Cesàro partial sums of harmonic series expansions...... 829

John L. Selfridge and Ernst Gabor Straus, On the determination of numbers by their sums of a fixed order ........................ 847

Annette Sinclair, A general solution for a class of approximation

problems .................................

George Szekeres and Amnon Jakimovski, $(C, \infty)$ and $(H, \infty)$ methods of summation...................................... 867

Hale Trotter, Approximation of semi-groups of operators. ............. 887

L. E. Ward, A fixed point theorem for multi-valued functions ........... 921

Roy Edwin Wild, On the number of lattice points in $x^{t}+y^{t}=n^{t / 2} \ldots \ldots .929$ 